\title{
Effects of smoking on thermal pain threshold in deprived and minimally-deprived habitual smokers
}

\author{
Paul Pauli', Harald Rau', Ping Zhuang', Stuart Brody', and Niels Birbaumer ${ }^{1.2}$ \\ ${ }^{1}$ University of Tübingen, Departement of Clinical and Physiological Psychology, Gartenstrasse 29, W-7400 Tübingen, Germany \\ ${ }^{2}$ Universtá degli Studi, Dipartimento Psicologia Generale, Piazza Cavour 23, I-35139 Padova, Italy
}

Received September 3, 1992 / Final version November 11, 1992

\begin{abstract}
This study examined the antinociceptive effects of smoking in nine habitual smokers under deprived $(12 \mathrm{~h})$ and minimally-deprived $(<30 \mathrm{~min})$ conditions. Pain threshold for thermal stimuli, heart rate, blood pressure and ratings of mood, arousal, dominance and well-being were assessed before and after smoking a cigarette. Overall, smoking affected all measured variables in the expected direction, leading to increased physiological activity, elevated pain threshold and improved mood. However, most of these effects depended on the deprivation status of the subjects, such that smoking after deprivation increased pain threshold whereas smoking after minimal deprivation did not. Pain threshold beforc smoking was the same for both groups. Deprived subjects had lower pre-smoke diastolic blood pressure, heart rate, and arousal levels, which rose to equal minimally-deprived subjects' scores after smoking.
\end{abstract}

Key words: Pain threshold - Smoking - Nicotine - Acute tolerance - Deprivation - Psychophysiological measures

The perception of pain seems to be modulated by nicotine intake. Antinociceptive effects of nicotine have been reliably shown in animals through the hot-plate and tail-flick methods (Phan et al. 1973; Tripathi et al. 1982; Aceto et al. 1983; Iwamoto 1989; Yang et al. 1992). However, studies in humans are rare and less conclusive. Several studies found no effects of smoking or nicotine intake on pain threshold or pain tolerance (Waller et al. 1983; Sult and Moss 1986; Knott 1990). Other results indicate that (a) deprived smokers exhibit lower pain threshold levels than nonsmokers or minimally-deprived smokers (Nesbitt 1973; Silverstein 1982) and that (b) smoking a nicotinecontaining cigarette leads to a pain threshold increase in smokers (Nesbitt 1973; Silverstein 1982; Pomerleau et al. 1984; Fertig et al. 1986).

Pomerleau and coworkers (Pomerleau et al. 1984; Fertig et al. 1986) also could demonstrate that the anti-

Correspondence to: P. Pauli nociceptive effect of nicotine occurs in minimally-deprived smokers. They argue that nicotine itself can produce direct relief from pain and that this reinforcement effect is independent of the state of nicotine withdrawal. However, they did not directly compare deprived and minimallydeprived smokers. Data regarding cardiovascular responses suggest larger effects of nicotine in deprived compared to minimally-deprived subjects (West and Russell 1987; Benowitz et al. 1990), an effect which can be interpreted as a sign of the development of acute tolerance (Porchet et al. 1988). Thus, it might be that the same cigarette has a stronger antinociceptive effect in deprived compared to minimally-deprived smokers. So far, no stud$y$ has explicitly compared pain thresholds in deprived and minimally-deprived smokers.

The present experiment used a within-subject design to evaluate the interaction between smoking deprivation and the effect of smoking on pain perception, physiological activity and mood. The following hypotheses were tested: (1) smoking increases pain threshold and arousal and improves mood; (2) these changes are especially pronounced after periods of smoking deprivation, leading to larger net effects than after periods of minimal deprivation.

\section{Materials and methods}

Subjects. Ten healthy, right-handed, male habitual smokers, all smoking more than ten cigarettes a day for more than 5 years, were studied. However, one subject was excluded because he failed to comply with smoking deprivation instructions. The remaining nine subjects were between 22 and 30 years old $(25.1 \pm 2.3)$, and had smoked between 12 and 27 cigarettes a day $(21.0 \pm 5.1)$ for 5-15 years $(8.0 \pm 3.0)$. Subjects were recruited through advertisements and were paid (DM 30) for participation. All were informed prior to the study that they would receive thermal stimuli at the pain threshold, and that they could terminate the experiment at any time without any negative consequences. All subjects signed a consent document.

Procedure, All subjects participated in two sessions. The difference between the sessions was that one followed a nicotine deprivation period of $12 \mathrm{~h}$, whereas subjects smoked a cigarette less than $30 \mathrm{~min}$ 
before the other session. The sequence of sessions was counterbalanced between subjects. Both sessions were carried out at the same time ( 9 or 10 a.m.) within 1 week. To ensure compliance, subjects were told that nicotine deprivation would be verified with a urine test, which was in fact not performed.

The dependent variables were pain threshold for thermal stimuli, systolic and diastolic blood pressure, heart rate, subjective wellbeing and subjective emotional state. Within each session pain thresholds were assessed before and after subjects smoked one cigarette of their usual brand. Blood pressure values and heart rate were measured before and after pain threshold determination, psychological measures only after pain threshold determination.

Smoking habits were assessed in the first session. After the experiment, subjects were asked about their expectancies regarding the influence of smoking and deprivation on pain perception.

Pain threshold determination Cutaneous heat stimuli were applied with the Path-Tester MPI-100 from Phywe Systeme GmbH Göttingen, Germany. The device controls a Marstock thermode that functions on the Peltier principle and can be both heated and cooled. The base temperature was $40^{\circ} \mathrm{C}$ and the rate of heating and cooling was set to $0.7^{\circ} \mathrm{C} / \mathrm{s}$. The contact surface of the thermode was $1.6 \times 3.6 \mathrm{~cm}^{2}$. Technical details, accuracy and reliability of the method are described by Galfe et al. (1990).

During the experiment the subjects sat in a comfortable armchair in front of a small table. The thermode came out through a square hole in the table. Subjects placed their left hand on the thermode, so that the thermode was in contact with the thenar of the hand. A spring kept the thermode pressed against the skin. The response panel was placed at the table, so that subjects could easily press the response key with the fingers of the right hand.

In each trial, the thermode was heated, and the subjects had to press the response button as soon as thcy perceived pain. The thermode was then actively cooled to the bascline temperature. This procedure was repeated eight times; so that eight threshold temperatures were determined. The mean of the last five trials was considered to be the actual pain threshold. The first three trials were run in order to adapt the subjects to the temperature of the thermode. Additionally, each session started with five practice trials. The intertrial interval was $10 \mathrm{~s}$. Each trial was announced by a beep, and the temperature started to rise after a (pseudorandomly determined) time period between 1 and $3 \mathrm{~s}$. The time for heating and cooling the thermode is dependent on the pain threshold of the subject (i.e. to reach $47^{\circ} \mathrm{C}$ it takes $2 \times 10 \mathrm{~s}$ to heat and cool the thermode; base temperature was always $40 \% \mathrm{C}$ and heating and cooling rate was $0.7^{\circ} \mathrm{C} / \mathrm{s}$ ), and therefore the actual trial length varied between subjects. On average, the eight pain stimuli were delivered within $4 \mathrm{~min}$.

Physiological measures. Blood pressure and heart rate were measured with an automated blood-pressure cuff. Measurements were conducted immediately before and after the pain threshold determination, leading to four values within each session.

Psychological measures. The psychological status of the subjects was assessed immediately after the pain threshold determination. Subjective well-being was measured with the Befindlichkeits-Skala (BFS) (von Zerssen 1976), a popular German questionnaire, which asks the subjects to describe their current well-being in terms of 28 pairs of adjectives, which together form a single factor of well-being. Subjects have to choose between three response categories, two of which are described by the adjectives, and the third indicating that neither adjective is relevant for the current state of well-being (e.g. Do you now feel: more relaxed, more tense or neither). Negative answers (e.g. tense) score two points and indifferent answers one point. The higher the score, the worse the subject currently feels.

The Self-Assessment Manikin (SAM, paper and pencil version; Lang 1980; see also Bradley et al. 1993) was used to assess the subjects' emotional state. The three dimensions "mood (or valence)", "arousal" and "dominance" were measured on nine-point scales, the different points represented by different manikins expressing the related emotional state. Higher values represent worse mood, higher arousal and less dominance.
Subjects' expectancies about pain threshold. Subjects' expectancies regarding the effect of smoking or deprivation on pain perception were assessed by the following two multiple choice questions at the end of the second session:

(1) When have you been more sensitive for thermal pain?

(a) Before smoking a cigarette.

(b) After smoking a cigarette.

(c) Smoking had no effect on pain sensitivity.

(2) On what experimental day have you been more sensitive for thermal pain?

(a) On the day when I was not allowed to smoke for $12 \mathrm{~b}$

(b) On the day when I smoked before the experiment.

(c) Pain sensitivity was equal on both days.

Data analysis. Repeated-measures analysis of variances (ANOVA) with the two within-factors Deprivation (deprivation versus no deprivation) and Smoking (pre-versus post-smoking) were conducted to analyse pain threshold and psychological data. To analyse the physiological measurements, the additional within-factor Painstimulus (pre- versus post-pain stimulus) was introduced. Significant effects were further evaluated with appropriate post-hoc means comparisons. Statistical analysis with the Order of sessions factor (first session deprived versus second session deprived) revealed no significant effects for this variable, and therefore these results will not be reported. $\mathrm{Chi}^{2}$-tests were used to analyse nominal data Level of significance was set at $5 \%$.

\section{Results}

\section{Pain threshold}

The ANOVA revealed significant Smoking $[F(1,8)=139$, $P=0.006]$ and Deprivation by Smoking $[F(1,8)=9.1, P$ $=0.02]$ effects (Fig. 1). Before smoking a cigarette, subjects showed about the same pain threshold in the deprived and the minimally-deprived condition. However, smoking had differential effects depending on the presmoke nicotine level. Smoking a cigarette after $12 \mathrm{~h}$ of deprivation led to a significant pain threshold increase of about $1^{\circ} \mathrm{C}$ (post-hoc comparison: $F=22.7, P=0.001$ ). On the other hand, smoking a cigarette under minimallydeprived conditions did not lead to pain threshold changes. This differential effect led to a significantly higher post-smoke threshold in the deprived compared to the minimally-deprived session ( $F=14.5, P=0.005$ ).

\section{Cardiovascular responses}

For diastolic blood pressure, the ANOVA indicated a marginally significant Pain-stimulus effect $[F(1,8)=4.8$, $P=0.08]$, a significant Smoking by Pain-stimulus interaction $[F(1,8)=6.1, P=0.04]$, and a highly significant Deprivation by Smoking interaction $[F(1,8)=13.0, P$ $=0.007]$. As can be inferred from Fig. 2, deprived subjects exhibited in the pre-smoke condition significantly lower diastolic blood pressure levels than minimallydeprived subjects $(F=26.0, P=0.0009)$. Smoking a cigarette was associated with significant blood pressure increases $(F=42.7, P=0.0002)$ in deprived subjects, whereas this effect did not reach significance in minimallydeprived subjects $(F=2.1, P=0.18)$. After smoking, both groups' diastolic blood pressure was practically identical. The significant Smoking by Pain-stimulus interaction can 


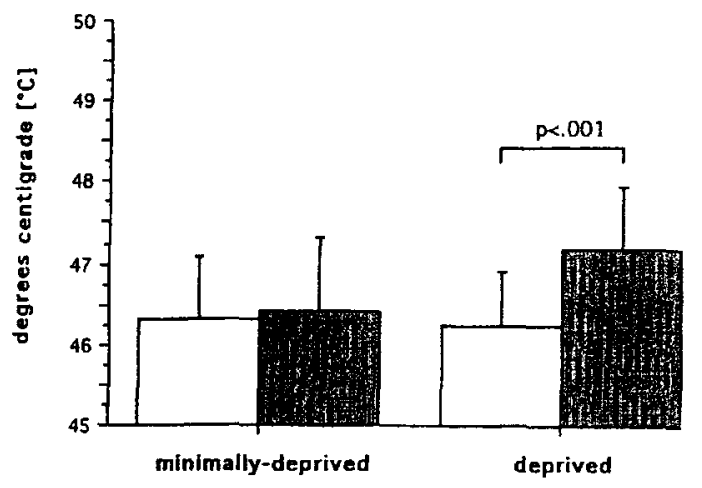

Fig. 1. Pain thresholds (means \pm SEMs; $N=9$ ) depending on deprivation status at the beginning of the experiment and smoking during the experiment. ( $\square$ ) Pre-smoke; (

be traced back to the fact that only the first post-smoke measurement indicated a significant higher diastolic blood pressure than in the pre-smoke condition $(F=22.8$, $P=0.001)$. The second post-smoke recording was not significantly different from the pre-smoke levels $(F=0.1$, $P=0.8$ ).

For systolic blood pressure, no significant main or interaction effects were found (Fig. 2).

The ANOVA for heart rate revealed highly significant Smoking $[F(1,8)=20.0, P=0.002]$, Deprivation by Smoking $[F(1,8)=17.7, P=0.003]$ and Smoking by Pain-stimulus $[F(1,8)=19.2, P=0.003]$ effects (Fig. 3). Post-hoc means comparisons for the Deprivation by Smoking effect mirrored the effects for diastolic blood pressure. Deprived smokers' heart rates were lower at the pre-smoke $(F=18.3, P=0.003)$ but equal at the postsmoke measurements $(F=2.8, P=0.13)$ compared to minimally-deprived smokers. The Smoking by Painstimulus interaction is related to the fact that heart rate, like diastolic blood pressure, decreased from the first to the second post-smoke measurements $(F=18.1, P$ $=0.003$ ), whereas similar changes between the two presmoke measurements did not occur. However, heart rate remained significantly higher in both post-smoke compared to the pre-smoke measurements $(F=65.0$, $P<0.0001 ; F=144.6, \quad P<0.0001)$, whereas diastolic blood pressure decreased to the pre-smoke level.

\section{Psychological responses}

Smoking a cigarette led to a significant improvement of mood [pre-smoke: $4.6 \pm 0.97$; post-smoke: $4.1 \pm 0.90$; $F(1,8)=6.0, P=0.04]$. Additionally, smoking led to a trend toward better subjective well-being [presmoke: $14.3 \pm 8.1$; post-smoke: $11.7 \pm 6.2 ; F(1,8)=3.6, P$ $=0.09$ ] and increased dominance [pre-smoke: $4.6 \pm 1.1$; post-smoke: $4.2 \pm 1.0 ; F(1,8)=4.0, P=0.08$ ). For arousal, a significant Deprivation by Smoking interaction was found $[F(1,8)=5.3, P=0.05]$. Under deprived conditions subjects' pre-smoke arousal was lower than under minimally-deprived conditions (pre-smoke: 3.1 \pm 1.1 versus $4.4 \pm 1.8 ; F=10.7, P=0.01$ ). However,
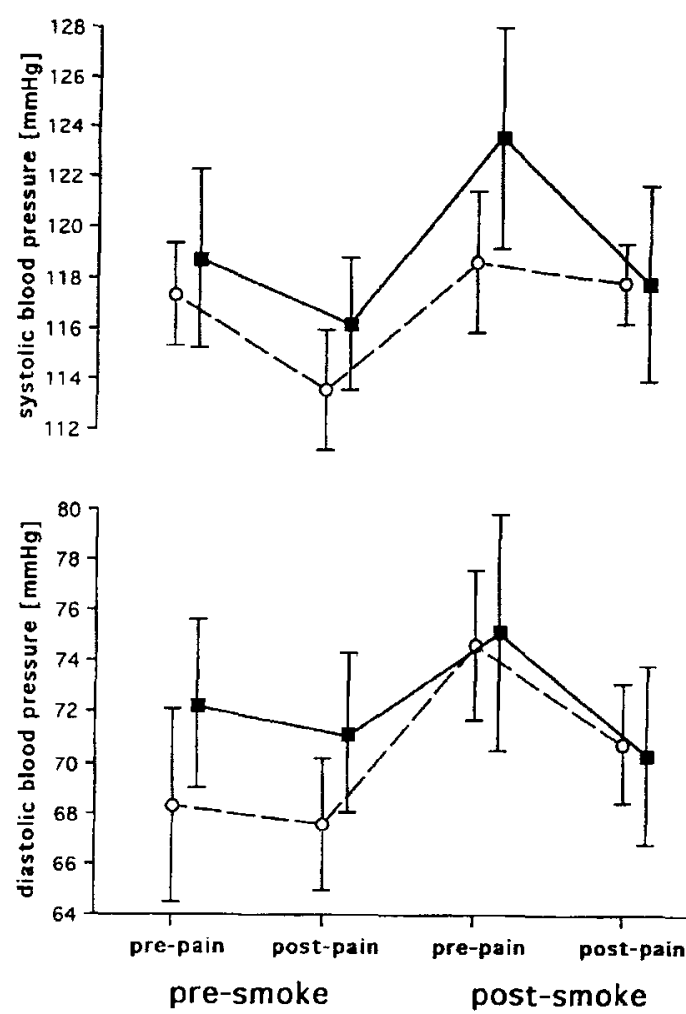

Fig. 2. Systolic and diastolic blood pressure (means $\pm S E M s$; $N=9$ ) depending on deprivation status at the beginning of the experiment and smoking during the experiment. (O) Deprived; (a) minimally-deprived

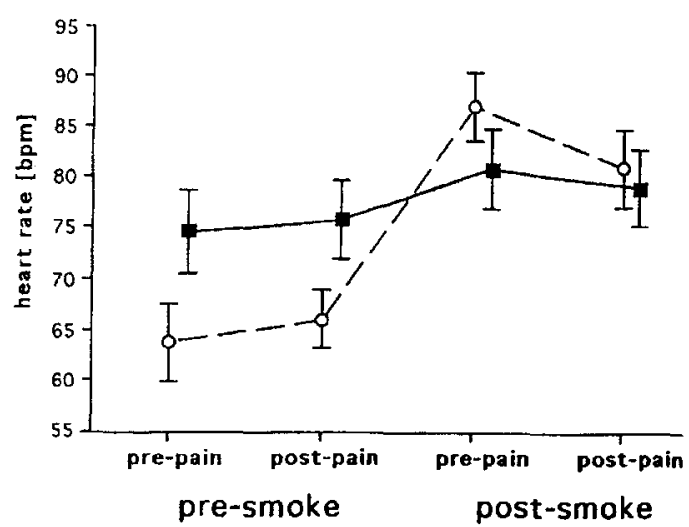

Fig. 3. Heart rate (means \pm SEMs; $N=9$ ) depending on deprivation status at the beginning of the experiment and smoking during the experiment. (O) Deprived; ( $D$ ) minimally-deprived

post-smoke arousal was practically the samc under both conditions (post-smoking: $3.8 \pm 1.2$ versus $3.8 \pm 1.9$ ), because smoking under deprived conditions led to an increase, whercas smoking under conditions of minimal deprivation led to a decrease in arousal. No other significant main or interaction effects were found for the psychological variables. 


\section{Expectancies}

There was no significant relationship between subjects' expectancies and objective pain threshold. Three subjects expected pain threshold to be lowest before smoking, two after smoking, and four were indifferent. However, only one subjects' pain threshold (mean for deprived and minimally-deprived conditions) was lower before compared to after smoking a cigarette $\left(\mathrm{Chi}^{2}=1.4, P=0.5\right)$. Regarding the effect of deprivation, two subjects expected to be more sensitive to pain stimuli after the deprivation period, three when they smoked before the experiment, and four were indifferent. For four subjects, these expectancies corresponded with the real pain thresholds on the 2 experimental days; however, the statistical test did not reach significance $\left(\mathrm{Chi}^{2}=2.2, P=0.3\right)$.

\section{Discussion}

Our results clearly indicate that the effect of smoking a cigarette on the psychological and physiological status of a subject depends on their deprivation status. There is an overall increase in heart rate and blood pressure, mood improvement, and elevation of pain threshold. However, the changes in pain threshold, diastolic blood pressure, heart rate and arousal were clearly more pronounced when subjects smoked a cigarette after: a deprivation period of $12 \mathrm{~h}$. Looking at baseline measures (pre-smoke), deprivation was associated with lower diastolic blood pressure, lower heart rate and lower subjective arousal, but not with lower pain thresholds or worse mood

This study confirms the previously observed interaction between deprivation status and heart rate changes due to smoking (West and Russell 1987; Benowitz 1990; Russell 1990). Additionally, similar effects were observed for changes in diastolic blood pressure. West and Russell (1987) found reports of subjective changes due to smoking only in deprived smokers, which would fit with our finding that smoking under deprived conditions leads to increased arousal. However, they found no calming effects of smoking, as we did under minimally-deprived conditions. West and Russell's question whether subjects had "felt anything from the cigarette" may not be entirely appropriate to assess calming effects. Pain threshold changes due to smoking also depend on the smoking deprivation status. In contrast to the cardiovascular and the arousal data, the deprivation had no effect on presmoke pain thresholds. The pre-smoke pain thresholds (under deprived and minimally deprived conditions) are in agreement with the normative data for normal subjects for the Path-Tester MPI- $100\left(46.3^{\circ} \mathrm{C}\right.$ versus $46.1^{\circ} \mathrm{C}$; Lautenbacher et al. 1989). Interestingly, no significant relationship between objective pain threshold changes and subjects' expectancies about the effect of smoking were found. The latter findings support the conclusion that the results reported above are physiological in nature and do not constitute expectancy-placebo effects.

At first glance, our data are at variance with results indicating that deprived smokers exhibit lower pain endurance levels than minimally-deprived smokers (Silverstein 1982), and that smoking a nicotine-containing cigarette leads to a pain threshold increase even in minim- ally-deprived smokers (Pomerleau et al. 1984; Fertig et al. 1986). The divergent results could be attributed to methodological differences regarding the pain stimuli (cold pressure, electrical stimuli, thermal pain), the control condition (no smoking, no-nicotine cigarette, pre-smoking measurement) and the experimental design (betweensubjects or within-subjects design). However, close scrutiny of prior studies reveals that these differences are not critical.

Silverstein (1982) compared smokers who were not allowed to smoke for the whole experiment (lasting about $1 \mathrm{~h})$ with smokers who smoked low- and high-nicotine cigarettes at the beginning of the experiment and during the delivery of electrical shocks (about $45 \mathrm{~min}$ later). Only the high-nicotine group differed from the other groups, and the nicotine-deprived and the low-nicotine group exhibited approximately the same pain threshold. Thus, no clear cut difference between deprived and minimallydeprived subjects was found. Additionally, it is not known when Silverstein's deprived subjects smoked their last cigarette.

Pomerleau et al. (1984) found even in minimally deprived smokers a higher pain threshold after smoking a nicotine-containing compared to a no-nicotine cigarette. However, as a consequence of the design, it was unclear whether the pain threshold increased due to smoking a nicotine-containing cigarette, or whether the pain threshold actually decreased as a consequence of the nonicotine cigarette Fertig et al. (1986) in fact found the lowest pain threshold in subjects performing sham smoking, somewhat higher thresholds in subjects smoking a zero-nicotine cigarette, and the highest pain thresholds in subjects smoking high-nicotine cigarettes or taking nicotine snuffs. It is possible that classically conditioned compensatory effects (Siegel 1983) or the frustration related to the zero-nicotine cigarette or the sham smoking procedure were responsible for the decrease in pain threshold.

Our study confirms acute tolerance for cardiovascular responses (heart rate and blood pressure), and demonstrates acute tolerance effects for pain perception. Porchet et al. (1988) developed a pharmacodynamic model of nicotine tolerance based on the empirical observation of heart rate changes following successive nicotine infusions separated by different time intervals, which may explain our results. Receptor down-regulation, presumably by a nicotine metabolite, is assumed to be the physiological basis for tolerance development.

In our data, smoking deprivation was not associated with reduced pain thresholds. This would fit with the approach of Pomerleau et al. (1984), who argue that the antinociceptive effect of nicotine is mediated by release of beta-endorphin, and that this effect is independent of the state of withdrawal. Following this assumption, our results suggest that smoking after a deprivation period is related to increased beta-endorphine release, or that betaendorphine under these circumstances has an especially powerful effect, or both processes may occur. It may be speculated that under conditions of nicotine deprivation fewer endorphine receptors are occupied, and therefore the released endorphine has more powerful effects. Our results are also consistent with the findings of West and Russell (1987) and others summarized in Warburton 
(1990), that acute tolerance and withdrawal effects of nicotine are found in some dimensions of behaviour, but not in others.

One limitation of this study is the lack of a direct measure of nicotine intake. West and Russell (1987) used a very similar design and found heart rate changes similar to ours, both under conditions of nicotine deprivation and under minimally deprived conditions. Additionally, West and Russell (1987) measured carbon monoxide (CO) as a measure of cigarette smoke inhaled over the preceding few hours. They found that the CO increase was somewhat greater after 24-h deprivation than during a normal smoking day. However, $\mathrm{CO}$ increase was significant under both conditions, and a considerable correlation $(r=0.53)$ between $\mathrm{CO}$ changes under the two conditions was found. Additionally, the post-cigarette $\mathrm{CO}$ after $24-\mathrm{h}$ abstinence was considerably lower than the pre-cigarette $\mathrm{CO}$ on the normal smoking day. Therefore, it seems unlikely that the differences we found between the deprived and minimally deprived conditions can be totally attributed to differences in nicotine intake or nicotine levels.

Acknowledgements. The authors wish to thank Michael Schwenzer for his valuable assistance. Rescarch was supported by the Deutsche Forschungsgemeinschaft (E1 103/3-2) and by NBN MedizinElektronik, München. The advice and discussion with Dr. Rahn and Dr. Röper from Reemtsma Research, Hamburg is also acknowledged.

\section{References}

Aceto MD, Awaya H, Martin BRM, May EL, (1983) Antinociceptive action of nicotine and its methiodide derivates in mice and rats. Br J Pharmacol 79:869-876

Benowitz NL, Porchet H, Jacob P (1990) Pharmacokinetics, metabolism, and pharmacodynamics of nicotine. In: Wannacott $S$, MAH Russell, IP Stolerman (eds) Nicotine psychopharmacology. Oxford University Press, Oxford, pp 112-156.

Bradley MM, Greenwald MK, Hamm AO (1993) Affective picture processing. In: Birbaumer N, A. Öhman (eds) The structure of cmotion. Hogrefe \& Huber, Seattle, pp 48-68

Fertig BJ, Pomerleau OF, Sanders B (1986) Nicotine-produced antinoception in minimally deprived smokers and ex-smokers. Addict Behav 11:239-248

Galfe G, Lautenbacher S, Hölzl R, Strian F (1990) Diagnosis of small-fibre neuropathy: computer assisted methods of combined pain and thermal sensitivity determination. Hospimedica $18: 38-48$

Iwamoto ET (1989) Antinociception after nicotine administration into the mesopontine tegmentum of rats: evidence for muscarinic actions. J Pharmacol Exp Ther 251:412 -421

Knott VJ (1990) Effects of cigarette smoking on subjective and brain evoked responses to electrical pain stimulation. Pharmacol Biochem Behav 35:341-346

Lang PJ (1980) Behavioral treatment and bio-behavioral assessment: computer applications. In: Sidowski JB, JH Johnson, TA Williams (eds) Technology in mental health care delivery systems Norwood, Ablex, NJ, pp 119-137

Lautenbacher S, Galfe G, Möltner A, Strian F (1989) Alterskorrigierte Normdaten für die Bestimmung der Temperatur-und Schmerzempfindlichkeit mit dem MPI-100 Path-Tester. MaxPlanck-Institut für Psychiatrie, München, unpublished manuscript

Nesbitt PD (1973) Smoking, physiological arousal, and emotional response. J Pers Soc Psychol 25[1]:137-144

Phan DV Dóda M, Bite A, György L (1973) Antinociceptive activity of nicotine. Acta Physiol Acad Sci Hung 44[1]:85-93

Pomerleau OF, Turk DC, Fertig JB (1984) The effects of cigarette smoking on pain and anxiety. Addict Behav 9:265-271

Porchet HC, Benowitz NL, Sheiner LB (1988) Pharmacodynamic model of tolerance: application to nicotine. J Pharmacol Exp Ther 244[1]:231-236

Russell MAH (1990) Nicotine intake and its control over smoking. In: Wonnacott S, MAH Russell, IP Stolerman (eds) Nicotine psychopharmacology. Oxford University Press, Oxford, pp 374-411

Siegel S (1983) Classical conditioning, drug tolerance, and drug dependence. In: Israel I (eds) Research advances in alcohol and drug problems, Plenum Press, New York

Silverstein B (1982) Cigarette smoking, nicotine addiction, and relaxation. J Pers Soc Psychol 42[5]:946-950

Sult SC, Moss RA (1986) The effects of cigarette smoking on the perception of electrical stimulation and cold pressor pain. Addict Behav 11:447-451

Tripathi HL, Martin BR, Aceto MD (1982) Nicotine-induced antinociception in rats and mice: correlation with nicotine brain levels. J Pharmacol Exp Ther 221[1]:91-96

von Zerssen D (1976) Die Befindlichkeits-Skala. Beltz. Test, Weinheim

Waller D, Schalling D, Levander S, Edman G (1983) Smoking, pain tolerance, and physiological activation. Psychopharmacology 79:193-198

Warburton DM (1990) Psychopharmacological aspects of nicotine. In: Wonnacott S, MAH Russell, IP Stolerman (eds) Nicotine psychopharmacology. Oxford University Press, Oxford, pp $77-111$

West RJ, Russell MAH (1987) Cardiovascular and subjective effects of smoking before and after $24 \mathrm{~h}$ of abstinence from cigarettes. Psychopharmacology 92:118-121

Yang C-Y, Wu, W-H Zbuzek VK (1992) Antinociceptive effect of chronic nicotine and nociceptive effect of its withdrawal measured by hot-plate and tail-flick in rats. Psychopharmacology $106: 417-420$ 